reference standard. Lymph nodes with macrometastases (the largest diameter $>2 \mathrm{~mm}$ ) were considered positive, while LNs with isolated tumour cells (ITC) and micrometastases (MIC) were considered negative.

Result(s)* 394 patients meeting the inclusion criteria between 2009 a 2019 were enrolled into the study. The characteristics of study population are shown in table 1 . Squamous cell carcinomas were most common (298/394) and the majority of cases was represented by early stage cancers (274/394), specifically IB1 FIGO 2009 (236/394). Macrometastes in pelvic LNs were pathologically confirmed in 53 patients $(13.5 \%)$ and micrometastases solely in 23 patients (5.8\%). Ultrasound failed to detect pelvic lymph node macrometastases in 15 patients $(3.8 \%)$ and median largest diameter of these unidentified metastases was $6 \mathrm{~mm}$ (range $3-11 \mathrm{~mm}$ ). There were 27 false positive ultrasound findings (6.9\%). Ultrasound reached sensitivity $71.7 \%$, specificity $92.1 \%$, PPV 58.5\%, NPV 95.4\% and overall accuracy $89.3 \%$.

Conclusion* Transvaginal/transrectal ultrasound is a reliable method for preoperative assessment of pelvic LNs in cervical cancer patients. It showed similar accuracy in detection of nodal macrometastases as reported for other imaging modalities. Similarly to all imaging methods, it had low sensitivity in detection of small volume macrometastases and micrometastases.

Key words: cervical cancer, lymph nodes, ultrasound, diagnostic accuracy

Klikně te nebo klepně te sem a zadejte text.

\section{THE UTILITY OF BIOMARKERS FOR OVARIAN CANCER RISK ASSESSMENT IN PRIMARY CARE: A PILOT STUDY}

${ }^{1} \mathrm{C}$ Barr*, ${ }^{2} \mathrm{G}$ Funston, ${ }^{3} \mathrm{D}$ Jeevan, ${ }^{3} \mathrm{SS}$ Sundar, ${ }^{4} \mathrm{LT}$ Mounce, ${ }^{5} \mathrm{E}$ Crosbie. ${ }^{1}$ Manchester University NHS FT, Division of Gynaecology, Manchester, UK; ${ }^{2}$ University of Cambridge, Department of Public Health and Primary Care, UK; ${ }^{3}$ University of Birmingham, Institute of Cancer and Genomic Sciences, UK; ${ }^{4}$ University of Exeter, Institute of Health Research, UK; ${ }^{5}$ The University of Manchester, Division of Cancer Sciences, UK

\subsection{6/ijgc-2021-ESG0.100}

Introduction/Background* Ovarian cancer is the leading cause of mortality from gynaecological malignancy. Survival improves with early diagnosis, however, early detection in primary care is challenging. The current blood test, cancer antigen 125 (CA125), has limited sensitivity and specificity for early disease. Human Epididymis 4 (HE4) is a promising diagnostic biomarker. We aimed to investigate the diagnostic accuracy and clinical utility of serum HE4 in a symptomatic primary care population.

Methodology We conducted a prospective observational study testing HE4 on primary care serum CA125 samples from women with suspected ovarian cancer in Manchester, UK, between April 2018 and April 2019. Serum HE4 was measured using chemiluminescent enzyme immunoassays following routine CA125 testing for clinical care. HE4 thresholds of $77 \mathrm{pmol} / \mathrm{L}$ and $150 \mathrm{pmol} / \mathrm{L}$ were used. The primary outcome was final diagnosis within 12 months of testing. Clinical outcomes were collected from hospital electronic patient records. Receiver operator characteristic (ROC) curves with area under the curve (AUC), sensitivity and specificity were calculated for CA125 and HE4 both alone and in combination. Age adjusted HE4 thresholds were calculated with linear regression models.
Result(s)* 1247 patients were included, with a mean age of 50 years (SD 15.7). 100 women had epithelial ovarian cancer; including 82 invasive and 18 borderline ovarian tumours. There was little difference in overall performance of CA125 and HE4 (AUC 0.932 vs 0.914 respectively). At a threshold of $77 \mathrm{pmol} / \mathrm{L}$, HE4 alone had a better sensitivity than CA125 [89\% (95\%CI 81.2-94.4) vs $81 \%(95 \%$ CI 71.9-88.2)] but a worse specificity $[75.6 \% \quad(95 \% \mathrm{CI} \quad 73-78)$ vs $92.2 \% \quad(95 \% \mathrm{CI}$ 90.4-93.6)]. HE4 and CA125 combined had improved sensitivity compared with CA125 alone (93\%, 95\%CI 86.1-97.1), but at a significant cost to specificity $(70 \%, 95 \% \mathrm{CI}$ 67.3-72.6). Serum HE4 levels were correlated with increasing age $(p$ $<0.001)$ and worsening eGFR $(\mathrm{p}<0.001)$. Age adjusted HE4 cut-offs marginally improved the specificity of CA125, however the numbers were small per age category and require validation in larger cohorts.

Conclusion* HE4 adds little to current diagnostic pathways in primary care. Age-adjusted thresholds may improve accuracy, but not sufficiently to recommend routine use at present.

\section{MYOMETRIAL INFILTRATION ASSESSMENT IN LOW-RISK ENDOMETRIAL CANCER BY 3D TRANSVAGINAL ULTRASOUND AND DIFFUSION-WEIGHTED MAGNETIC RESONANCE IMAGING}

${ }^{1} \mathrm{~N}$ Carreras Diéguez*, ${ }^{2} \mathrm{M}$ Munmany, ${ }^{3} \mathrm{C}$ De Guirior, ${ }^{1} \mathrm{P}$ Fusté, ${ }^{3} \mathrm{I}$ Matas, ${ }^{1} \mathrm{~B}$ Diaz-Feijoo, ${ }^{1} \mathrm{AG}$ Glickman, ${ }^{4} \mathrm{~L}$ Buñesch, ${ }^{1} \mathrm{~N}$ Agustí, ${ }^{3} \mathrm{C}$ Ros, ${ }^{4} \mathrm{M}$ Sebastià, ${ }^{1} \mathrm{M}$ Del Pino, ${ }^{5} \mathrm{~A}$ Saco, ${ }^{4} \mathrm{C}$ Nicolau, ${ }^{5} \mathrm{~J}$ Ordi, ${ }^{1} \mathrm{~A}$ Torne. ${ }^{1} \mathrm{Hospital}$ Clínic de Barcelona, Gynaecologic Oncology Unit, Barcelona, Spain; ${ }^{2}$ Hospital Clínic de Barcelona, Gynaecology, Obstetrics and Neonatology, Barcelona, Spain; ${ }^{3}$ Hospital Clínic de Barcelona, Gynaecology, Obstetrics and Neonatology, Barcelona, Spain; ${ }^{4}$ Hospital Clínic de Barcelona, Radiology, Barcelona, Spain; ${ }^{5}$ Hospital Clínic de Barcelona, Pathology Department, Barcelona, Spain

\subsection{6/ijgc-2021-ESG0.101}

Introduction/Background* In patients with early-stage, grade 12, endometrioid endometrial cancer, preoperative assessment of myometrial invasion is essential to define the need of pelvic and paraaortic lymph node dissection. Our aim was to evaluate the role of three-dimensional transvaginal ultrasound (3D-TVUS) and diffusion-weighted magnetic resonance imaging (DW-RMI) for the assessment of myometrial infiltration in patients with well-differentiated (G1) or moderately differentiated (G2) endometrioid endometrial carcinoma (EC).

Methodology We performed a retrospective observational study. Myometrial infiltration was assessed by 3D-TVUS and DW-MRI in 152 women with G1 or G2 endometroid EC who underwent surgical treatment in a tertiary referral center between 2012 and 2019. Sensitivity, specificity, predictive values and accuracy for the two techniques and for a combination of both were computed. Definitive histopathological data in the surgical specimen regarding myometrial infiltration was used as 'Gold Standard'.

Result(s)* One hundred and fifty-two patients were included, $120(79 \%)$ patients presented myometrial infiltration $<50 \%$ in postoperative analysis of surgical specimen and $32(21 \%)$ patients presented deep myometrial infiltration (>50\%). 3DTVUS and DW-MRI showed an agreement of $78.9 \%$ with a kappa index of 0.44 for the detection of deep myometrial infiltration. Sensitivity, specificity and accuracy of 3D-TVUS for the detection of deep myometrial infiltration were $71.0 \%$, $80.5 \%$ and $78.5 \%$ respectively. Evaluation of myometrial 
infiltration with DW-MRI had a sensitivity, specificity and accuracy of $76.2 \%, 84.4 \%$ and $82.9 \%$ respectively. Association of both techniques increased sensitivity and specificity up to $84.6 \%$ and $93.2 \%$ and provided a low false negative rate $(2.3 \%)$.

Conclusion* The combination of 3D-TVUS and DW-RMI offers a high sensitivity and specificity to identify deep myometrial infiltration in patients with endometrioid G1 or G2 EC, thus these patients might benefit from performance of both techniques in preoperative evaluation.

\section{THE SIGNIFICANCE OF SINGLE VERSUS MULTIPLE POLYPS AT HYSTEROSCOPY IN POST MENOPAUSAL BLEEDING}

J Davies, L Honeyman, K Hartshorn, J Dasgupta, A Phillips, S Kolhe. Derby Gynaecological Cancer Centre, Derby, UK

\subsection{6/ijgc-2021-ESGO.102}

Introduction/Background* The significance of endometrial polyps in women presenting with post menopausal bleeding (PMB) is uncertain, with variable risk of malignancy between $3.5 \%$ and $6 \%$. Outpatient hysteroscopy is now the standard of care for endometrial assessment with hysteroscopic polypectomy is increasingly performed in this setting. We aimed to establish the relevance and malignancy risk in women with solitary or multiple polyps presenting with PMB.

Methodology A retrospective review of prospectively recorded data between October 2017 and December 2019; during which 449 patients underwent outpatient hysteroscopy and polypectomy for bleeding. Records were interrogated for patient, procedural and histological factors.

Result(s)* The mean age of patients undergoing polypectomy was 63 [IQR 55-70] years. Of these only 69/449 (15\%) did not have polyps detected on ultrasound prior to hysteroscopy. Vaginoscopic hysteroscopy was attempted in 398 cases was successful in 359 (90\%) of cases. Quality of assessment was determined by stated visualization of both ostia, this was achieved in $400(89 \%)$ cases. Only 98 cases (21\%) were described as difficult of which the main causes were cervical stenosis, cervical tortuosity or uterine lie.

For patients undergoing a single polypectomy $(n=286) .276$ cases were benign, 9 had hyperplasia without atypia, 7 had hyperplasia with atypia and 17 had cancers with one sample insufficient.

For patients undergoing multiple polypectomy $(n=162)$. 137 cases were benign, 10 had hyperplasia without atypia, 7 had hyperplasia with atypia and cancer was diagnosed in 21 .

The rate of cancer in multiple polyps and single polyps was $13 \%$ and $6 \%$ respectively, with multiple polyps conveying a $2.04 \mathrm{x}$ increased risk of malignancy.

Conclusion* Outpatient polypectomy is a safe and well tolerated procedure with low complication rates. Multiple polyps can be resected in outpatient see-and-treat setting and should be encouraged due to the increased rates of cancer seen in those with multiple polyps.

\section{TRANSVAGINAL ULTRASOUND-GUIDED CORE BIOPSY - OUR EXPERIENCES IN A COMPREHENSIVE CANCER CENTRE}

1;2D Lengyel, 1;2Z Novák, ${ }^{3} \mathrm{~K}$ Kőhalmy, ${ }^{4}$ I Vereczkey. ${ }^{1}$ University of Szeged - Faculty of Medicine, Doctoral School of Clinical Medicine, Szeged, Hungary; ${ }^{2}$ National Institute of Oncology, Department of Gynaecology, Budapest, Hungary; ${ }^{3}$ National Institute of Oncology, Department of Biochemistry, Budapest, Hungary; ${ }^{4}$ National Institute of Oncology, Department of Surgical and Molecular Pathology, Budapest, Hungary

\subsection{6/ijgc-2021-ESGO.103}

Introduction/Background" Histological diagnosis of female pelvic tumours is essential in their adequate and early clinical management. Ultrasound-guided biopsy is a routine diagnostic method to obtain tissue samples and used widely in different types of tumours. Pelvic solid masses can be biopsied via transabdominal, transvaginal, transrectal or transperineal routes. Generally, lesions located deep within the female pelvis are not easy to access transdabdominally due to various bowel loops, major vessels, uterus, urinary bladder and ureter being in the path of the needle.

Methodology We report our experience of transvaginal ultrasound (TVUS)-guided core biopsies involving 303 patients referred to the gynaecological ultrasound unit of our national comprehensive cancer centre. All patients who underwent a transvaginal, ultrasound-guided core needle biopsy sampling between March 2019 and December 2020 were included.

Result(s)* Adequate histologic specimens were obtained in 299 patients (98.7\%). The most common sites of biopsy sampling were the adnexa (29.7\%), the vaginal stump or wall $(13.5 \%)$, the uterus $(11.6 \%)$ and the peritoneum (10.2\%). Malignancy was confirmed in two-thirds of patients (201/303) and a primary malignancy was diagnosed in 111 of the 201 histologically verified malignant cases (55.2\%). Interestingly, 23.9\% (48/201) of malignant tumours were proven to have a non-gynaecological origin. Among them, gastrointestinal tumours occurred the most frequently (31/48 patients). Three abscesses were discovered following the biopsy procedure, resulting in a complication rate of $1 \%$. In $94(31 \%)$ patients, subsequent surgery allowed the comparison of the ultrasound-guided and surgically obtained histologic results. We found inaccuracy in 12 cases (12.8\%), which is discussed in this paper in detail. Sensitivity, specificity, PPV and NPV to diagnose malignancy was $94.8 \%$, 94.1\%, 98.7\% and 80.0\%, respectively.

Conclusion* According to our experience, TVUS-guided NCB is a safe and effective histological sampling procedure, providing adequate tissue for pathological evaluation in $99 \%$ of cases. It can reliably guide therapy as its performance is satisfactory compared to surgically obtained histology. As infectious complications might rarely occur, routine preoperative vaginal disinfection is suggested. In case of the suspicion of malignancy despite negative biopsy histology, further investigation is proposed due to the $80 \%$ NPV.

\section{BONE SCAN IN GYNECOLOGICAL CANCER}

CF Ghomari*, A Medjahedi. Nuclear Medicine, Tlemcen University Hospital. Dr Tidjani Damerdji; Tlemcen University, Medicine Departement; Tlemcen, Algeria

10.1136/ijgc-2021-ESG0.104

Introduction/Background* Bone metastases of gynecological cancer are rare. To complete the initial staging of the disease, 